\section{GENRE}

en séries
Genre en séries

Cinéma, télévision, médias

$11 \mid 2020$

Freaks en tous genres : corps mutants, cyborgs, métamorphoses \& fantastiques

\title{
Haïr et railler les femmes en ligne : une revue de la littérature sur les manifestations de cyber misogynie
}

Delphine Dupré et Valérie Carayol

\section{(2) OpenEdition}

\section{Journals}

Édition électronique

URL : http://journals.openedition.org/ges/1072

DOI : $10.4000 /$ ges.1072

ISSN : 2437-6563

Éditeur

Presses universitaires de Bordeaux

Référence électronique

Delphine Dupré et Valérie Carayol, « Haïr et railler les femmes en ligne : une revue de la littérature sur les manifestations de cyber misogynie », Genre en séries [En ligne], 11 | 2020, mis en ligne le 01 juin 2020, consulté le 18 février 2021. URL : http://journals.openedition.org/ges/1072 ; DOI : https:// doi.org/10.4000/ges.1072

Ce document a été généré automatiquement le 18 février 2021.

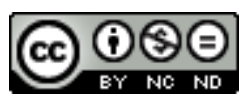

La revue Genre en séries est mise à disposition selon les termes de la Licence Creative Commons Attribution - Pas d'Utilisation Commerciale - Pas de Modification 4.0 International. 


\title{
Haïr et railler les femmes en ligne : une revue de la littérature sur les manifestations de cyber misogynie
}

\author{
Delphine Dupré et Valérie Carayol
}

\section{Introduction}

1 Cet article vise à documenter et analyser le phénomène de la misogynie en ligne à partir d'un tour d'horizon des travaux scientifiques, issus de la littérature française et anglo-saxonne, publiés récemment sur ce sujet.

Dans la recherche anglo-saxonne, la question de l'émergence de formes de violences genrées dans le cyber espace s'est posée dès le début de la démocratisation d'Internet. Dès 1996, McCormick et Leonard observent l'ambiance très « masculine » qui règne sur le Web et s'interrogent sur ses conséquences sur la participation des femmes. En 2005, Azy Barak entreprend la première revue de la littérature sur le harcèlement sexuel en ligne. Dans la littérature française, Josiane Jouët publie, en 2003, un article de synthèse sur le rôle des TIC dans la construction des identités genrées et le développement de nouveaux rapports de genre façonnés par ces technologies.

3 La crise dite du \#Gamergate, amorcée sur Twitter en 2014, constitue l'un des évènements qui a suscité l'intérêt des chercheurs anglo-saxons en communication (Jane, 2016). Le point de départ du Gamergate est un message posté sur un forum de discussion par un développeur de jeux vidéo, Eron Gjoni, accusant faussement son ex-compagne, Zoe Quinn, également conceptrice de jeux vidéo, d'avoir eu des relations intimes avec un journaliste pour accroître la popularité de son jeu intitulé Depression Quest. Sous le prétexte de lutter contre les interférences entre journalistes et concepteurs, de nombreux internautes ont entamé, de manière anonyme, une campagne de haine et de harcèlement envers plusieurs figures de proue féminines de la scène du jeu vidéo. Ce harcèlement s'est rapidement étendu à toute personne - journaliste, personnalité publique, etc. - cherchant à apporter son soutien aux victimes (Mortensen, 2016). La 
violence du Gamergate, qui a contraint certaines victimes à cesser leur activité professionnelle en lien avec le jeu vidéo, a mis en exergue l'étendue de la misogynie qui sévit impunément sur le web. La présence, dans les rangs des instigateurs, de sympathisants d'extrême droite, a révélé les liens étroits entre haine misogyne et propagande politique.

4 Plusieurs travaux, dont nous souhaitons rendre compte dans cet article, ont été publiés à la suite de cet évènement. Ils visent à mieux comprendre les actes misogynes perpétrés sur les principaux réseaux socionumériques (Twitter, Facebook, Instagram, etc.), sur les forums de discussion, ou encore sur des sites plus confidentiels, comme Reddit ou 4chan, au sein desquels règnent un entre-soi et une culture, souvent qualifiée de " toxique », qui favoriseraient des expressions de haine décomplexées.

5 En France, les recherches sur cette thématique portent principalement sur les dispositifs "techno-sémiotiques» des plateformes numériques qui exacerbent les conflits entre acteurs revendiquant différentes conceptions du genre (Julliard, 2014, 2016, 2017; Cervulle et Julliard, 2018; Quemener, 2018) ainsi que sur les dimensions genrées du cyber harcèlement (Couchot-Schiex et Moignard, 2016 ; Blaya 2018, 2019).

6 Plusieurs concepts désignant des formes de violence survenant dans le cyber espace et ciblant principalement des femmes, sont disponibles dans la littérature scientifique: cyber harcèlement, cyber violence genrée, violence sexiste en ligne, gendertrolling, etc. Dans cet article, nous utiliserons le terme plus englobant de " cyber misogynie ».

7 Nous nous attèlerons, à partir d'une étude de la littérature mettant en regard des corpus de travaux sur la misogynie et sur les violences numériques à répondre à la question suivante: quelles caractéristiques de la misogynie en ligne sont mises en lumière par la littérature scientifique récente?

8 Les textes scientifiques qui composent cette revue de la littérature ont été recueillis à partir des mots clés «misogynie en ligne », «cyber violence sexiste » ainsi que «cyber misogyny ", "cyber sexism " et "cyber gendered hate». Le corpus qui sert de support à notre analyse est constitué d'un ensemble de textes, parus entre 1996 et 2018, en français et en anglais. Les 70 articles qui composent notre corpus ont été collectés principalement sur le portail de revues Cairn, ainsi que sur les bases de données Sage Publications et Taylor \& Francis. Feminist media studies est, dans notre corpus, la revue la plus pourvoyeuse d'articles sur la misogynie en ligne.

9 Les terrains investigués dans les articles de notre corpus varient en fonction du phénomène étudié. Le cyber harcèlement entre jeunes est analysé au moyen de questionnaires, de focus groups et d'entretiens semi-directifs auprès d'échantillons de collégien-e-s, lycéen-e-s et étudiant-e-s de l'enseignement supérieur. Les campagnes de dénigrement ciblant de personnalités médiatiques ou politiques font l'objet d'études de cas à partir de sources documentaires (articles de presses, archives judiciaires), de traces numériques issues des réseaux sociaux et d'entretiens avec les victimes. Enfin, plusieurs recherches sur les manifestations de haine sur les réseaux sociaux, les sections commentaires des sites de presse ou les forums de discussion ont été entreprises via la collecte et le traitement des messages postés sur ces plateformes.

10 Dans un premier temps, nous étudierons les différentes manières dont les fonctionnalités techniques du web permettent de matérialiser et d'exprimer un certain nombre d'affects négatifs envers les femmes. Dans un deuxième temps, nous analyserons les différents acteurs mis en mouvement par les affects négatifs 
préalablement identifiés. Enfin, nous présenterons les dimensions financières et juridiques de la cyber misogynie.

\section{Manifestations et matérialisation des affects négatifs associés à la misogynie}

11 S'inspirant des recherches menées dans les années 1970 par Albert Mehrabian sur l'importance de la communication non-verbale, un certain nombre de travaux issus de la littérature sur la communication médiatisée par ordinateur (Sproull et Kiesler, 1986 ; Culnan et Markus, 1987) postulent que l'absence de langage para-verbal (gestes, mimiques faciales, postures, etc.) et d'indices contextuels produirait une communication numérique "pauvre", source de malentendus et de conflits. Ces travaux, focalisés principalement sur les incidences d'un mode de communication basé sur l'écrit, constituent à ce jour un cadre de référence pour analyser les interactions médiatisées par ordinateur, tant en contexte de travail que dans l'espace public numérique.

Les résultats de ce courant de recherche, qui a pu être qualifié de «déterminisme technologique " (Markus, 1994), tendent à suggérer que la communication numérique ne permet pas de véhiculer des émotions. Ces recherches ont toutefois été nuancées par les travaux des années 1990-2000 sur les phénomènes de flaming sur Internet (Friedman et Currall, 2003 ; Krohn, 2004) ainsi que ceux plus récents sur le web affectif (GardeHansen et Gorton, 2013 ; Allard et al., 2017 ; Alloing et Pierre, 2017) qui ont démontré que les caractéristiques du web offraient de multiples ressources pour exprimer, matérialiser et monétiser l'agressivité, le mépris ou le ridicule.

Dans le cadre de son travail doctoral, Ranida Boonthanom (2004) a montré que lors de conversations médiatisées par ordinateur, les émotions des interlocuteurs étaient véhiculées par des "indices para-verbaux» et des "indices verbaux" dits "de substitution ». La première catégorie fait référence au «vocal spelling» correspondant aux onomatopées (Ex: Oooh!») Les émotions peuvent également être exprimées via l'usage d'émoticons. Enfin, l'auteure évoque les "marqueurs grammaticaux ", c'est-àdire, le fait de rédiger des messages avec des mots en gras, en majuscules et de nombreux points d'exclamation.

Les «indices verbaux» correspondent aux mots ou abréviations désignant des émotions (joie, tristesse, colère etc.) et aux « marqueurs linguistiques », c'est-à-dire aux formules qui permettent d'exprimer des émotions sans les nommer (2004, p. 21). Pfaff et Pirzadeh (2017) ajoutent que ces marqueurs linguistiques peuvent être des hyperboles ou des euphémismes. Plusieurs études quantitatives et qualitatives révèlent que ces ressources numériques sont mobilisées pour exprimer un continuum d'émotions négatives prenant les femmes pour objet - au travers de détournements parodiques, d'insultes, de menaces, etc. -, qui s'étendent de l'amusement à la haine (Megarry, 2014 ; Bertini, 2012 et 2016 ; Hardaker et McGlashan, 2016 ; Jane, 2016).

\section{L'humour misogyne : des poncifs traditionnels à la violence ambiguë}

Jessica Drakett et ses collègues (2018) étudient les mèmes ${ }^{1}$ humoristiques, élaborés principalement par les tenants de la culture geek, qui circulent librement sur le web. 
Les auteures observent que ces mèmes s'appuient sur la force de l'image, exacerbée par le texte d'accompagnement, pour reconduire, sous couvert d'humour, un ensemble de normes de comportement hétéronormatives (hommes sexuellement actifs versus femmes passives) et de poncifs relatifs aux traits de caractère dévalorisants attribués traditionnellement au féminin (passivité, irrationalité, frivolité, etc.).

Marie-Joseph Bertini $(2012 ; 2016)$ a étudié les réactions des internautes sur Twitter face à certains évènements médiatiques, dont «l'affaire DSK ». Elle observe le nombre important de tweets sarcastiques et railleurs qui véhiculent des stéréotypes de genre éculés et des croyances misogynes archaïques qui ne seraient probablement plus tolérées dans l'espace public hors ligne. Elle en conclut que le web instaurerait une «nouvelle forme de ritualisation des interactions sociales et langagières fondée sur la dérision, le cynisme et le détournement sardonique (railleur et sarcastique)» (2016 : 38) défavorable aux femmes.

17 Ce constat d'un retour ou, du moins, d'une visibilité inédite d'anciennes formes de misogynie sur le web est partagé par Maurice Daumas (2017: 110) : «les devinettes et plaisanteries misogynes qui circulent sur Internet sont de la même nature que celles des siècles passés ». La mise en perspective historique réalisée par l'auteur révèle le rôle "socialisant" du rire misogyne, dans la mesure où "l'humour concourt à la perpétuelle reconstruction d'un 'nous'. Mais s'il y a un 'nous', c'est qu'il y a un 'eux' (qui est ici un 'elles'), contre lequel on bataille. Cultiver l'entre-soi, c'est construire un adversaire à surmonter. » (112)

Le rire misogyne comme rituel de camaraderie virile a également été mis en exergue dans les travaux de Walter Dekeseredy et Martin Schwartz (2016) dans le cadre de leur théorie du soutien social des pairs masculins (male peer support theory) qu'ils appliquent comme cadre de référence à l'étude des abus sexuels en ligne. La dérision des femmes sur le web, moquées en tant qu'objets sexuels et/ou potiches écervelées, s'inscrit dans le prolongement de rituels de sociabilité circonscrits autrefois dans des espaces-temps quasi exclusivement masculins (les bars, les vestiaires, les clubs de football, certains milieux professionnels, etc.), toutefois Internet accroît la visibilité ainsi que l'audience de ce rire masculin.

Les auteurs précisent que les pratiques consistant à échanger et visionner du matériel pornographique entre hommes afin de se divertir et, ainsi, de consolider des liens fraternels, ont été documentées dans la littérature sociologique sur les masculinités (Kimmel, 2009). Les nombreuses possibilités de partage de photos et de vidéos intimes de femmes sur les plateformes numériques s'inscriraient, d'après Dekeseredy et Schwartz, dans la continuité des activités propres à certaines formes de socialisation masculines qui valorisent et perpétuent un ensemble de croyances et d'attitudes qualifiées de « patriarcales ».

Sur ce point, les auteurs établissent une filiation entre les pratiques « récréatives » et socialisantes basées sur le partage de matériel pornographique et la reconfiguration de l'industrie du porno à l'ère numérique. Le numérique a contribué, d'une part, à la démocratisation de productions " amatrices » dont le succès a conduit à l'adoption de ce format par les professionnels du secteur. La deuxième tendance observée par les auteurs est la prolifération de contenus, en abondance et facilement accessibles, représentant des pratiques sexuelles violentes envers les protagonistes féminins (dont le consentement peut parfois paraître douteux). Ces deux évolutions simultanées ont participé à la naturalisation et la banalisation des abus sexuels en ligne. 
la diffusion de vidéos de violences sexuelles sur Internet, notamment des viols, s'accompagne fréquemment de messages moqueurs et de nombreux «lol». Ainsi, le partage de photos et de vidéos d'abus sexuels tend à devenir un divertissement dont les victimes, déshumanisées, sont tournées en dérision.

Outre son rôle socialisant, ce rire misogyne tend à être favorisé par les fonctionnalités techno-discursives des plateformes numériques (Julliard, 2014; 2016). Ainsi, la «ludification » de la communication sur les réseaux socionumériques, inscrite dans le design des dispositifs techniques de ces plateformes, a été amplement documentée (Frame, 2017 ; Mercier, 2018). Or, cette dimension ludique, qui procède de la possibilité d'obtenir des likes, des retweets, de réaliser des montages parodiques, a conféré un aspect "récréatif " aux actes misogynes perpétrés en ligne et a contribué à leur surenchère (Salter, 2017). Alexander Frame précise que « derrière la "culture du Lol" se cachent aussi des agressions d'une grande violence symbolique (...)» (2017: 194). L'humour misogyne serait ainsi mobilisé pour exprimer, sous couvert de second degré, des violences sexuelles et psychologiques particulièrement dérangeantes. À titre d'exemple, la pratique consistant à associer des smileys connotant une émotion positive ou des onomatopées du type "ahahah" à des menaces de viol crée de la confusion et permet de contourner les règles de modération des plateformes, en modifiant notamment la «tonalité » du message (Cole, 2015; Drakett et al., 2018 ; Benton-Greig et al., 2018; Thompson, 2018).

Il ressort de ces travaux que les formes de misogynie exprimées au travers de l'humour pourraient être positionnées sur un continuum, en fonction de leur intensité. Ce continuum irait des moqueries basées sur des stéréotypes de genre à des violences symboliques plus pernicieuses et des abus sexuels banalisés en raison de leur abondance et de leur accessibilité.

24 Ainsi, les différentes formes de dérision, de sarcasme et d'humour misogynes se fonderaient sur les ressources du web pour véhiculer une conception essentialiste et hiérarchisée des identités et des rapports de genre tout en neutralisant la critique et la censure potentielle des plateformes (Dragiewicz et al., 2018). Le fait que ces contenus humoristiques ne soient pas modérés pose problème. En effet, Claire Hardaker et Mark McGlashan (2016) ont réalisé une enquête quantitative sur Twitter et ont observé un effet de spirale. Ils ont découvert que les insultes misogynes les plus graves résultaient d'une escalade de violence, d'un processus de galvanisation des internautes souvent déclenché par des tweets ironiques ou sarcastiques. Ainsi, les catégories de l'humour et de l'insulte misogynes en ligne semblent étroitement liées. L'identification d'un bouc émissaire (Girard, 1982), semble servir dans les deux cas la constitution d'un groupe (Daudigeos, 2014) prêt à des lynchages numériques.

\section{Les expressions de haine dans la communication dyadique et groupale}

25 Un ensemble de travaux, issus de la littérature française et anglophone, porte sur les expressions de haine misogyne rendues possibles tant par les TIC que par les spécificités des plateformes numériques. Ces phénomènes, ancrés dans des dynamiques sexistes, homophobes et racistes, sont analysés dans le cadre d'études sur la communication dyadique et sur la communication groupale.

Genre en séries, 11 | 2020 


\section{Le cyber harcèlement} sociales qui lui préexistent. Les différentes formes de brimades numériques, initiées dans une interaction dyadique puis relayées par plusieurs internautes, peuvent constituer une manière de sanctionner des comportements qui s'écartent des normes hétérosexuelles traditionnelles (Myers et Cowie, 2017). Dans cette veine, les personnes LGBT courent davantage de risques que leurs camarades hétérosexuel-le-s d'être la cible de cyber harcèlement (Shariff et Gouin, 2006; Felmlee et Faris, 2016). Cela est particulièrement vrai pour les étudiant-e-s, dont certain-e-s habitent loin de leurs parents pour la première fois et s'autorisent, de ce fait, à expérimenter des relations queer.

\section{Le revenge porn}

Un certain nombre de travaux issus de notre corpus s'intéresse au phénomène qualifié de revenge porn qui désigne la publication et la diffusion non consensuelle de contenus (photos, vidéos, montages, etc.) sexuellement explicites. À l'instar du cyber harcèlement, il s'agit d'un phénomène genré dans la mesure où les victimes sont, dans leur vaste majorité, des femmes (Sebastian, 2017 ; Patella-Rey, 2018). Suivant la même logique, les instigateurs tendent à être principalement des hommes (Dekeseredy et Schwartz, 2016).

Précisons, de prime abord, que le terme "revenge porn " a fait l'objet de nombreuses critiques. Parler de « revanche » s'avère psychologisant et réducteur. Cette désignation focalise l'attention sur le mobile présumé de l'instigateur et occulte les différentes motivations qui peuvent inciter les internautes à perpétrer de tels actes (pour se 
divertir entre amis, pour monétiser des contenus sexuels sur certaines plateformes, pour accroître leur notoriété, etc.). De plus, ce terme a l'inconvénient de susciter une relation d'empathie avec l'agresseur, en véhiculant implicitement l'image d'un homme éconduit cherchant à se venger par douleur. Adopter ainsi le point de vue de l'instigateur conduit à minimiser les nombreuses conséquences délétères - en termes de santé physique et psychique, de réputation professionnelle, etc. - auxquelles les victimes doivent faire face.

31 Les instigateurs peuvent acquérir des photos et vidéos intimes de plusieurs manières (McGlynn et al., 2017; Ricciardelli et Adorjan, 2018). Elles peuvent tout d'abord leur avoir été transmises spontanément par les victimes ou sous le coup de la contrainte (en raison d'un chantage, par exemple). Ces contenus peuvent résulter de montages, qui consistent à superposer le visage de la victime sur un corps dénudé, réalisés via Photoshop ou par des sites spécialisés. Ces contenus peuvent également avoir été extorqués aux victimes. Le cas du piratage du Cloud de l'actrice Jennifer Lawrence et du vol de ses photos intimes constitue un exemple emblématique de cette pratique. Enfin, et dans le pire des cas, ces contenus sexuellement explicites peuvent avoir pour origine l'immortalisation d'un viol via un téléphone portable ou tout autre support d'enregistrement.

Les femmes dont les photos intimes circulent sur Internet sont réprimandées pour leur imprudence, leur irresponsabilité. Les critiques à leur encontre font ainsi peu de cas de la malveillance et du manque de considération dont font preuve les instigateurs. Les auteur-e-s (Mantziari, 2017 ; Patella-Rey, 2018) reconnaissent ici la tendance à blâmer la victime pour la violence masculine subie et à sanctionner les femmes qui ne se conforment pas aux normes de comportements hétérosexuelles contraignantes (tempérance, réserve, etc.) Ces croyances contribuent à alimenter une «culture du viol » qui véhicule l'idée que, d'une certaine manière, en raison de leur attitude provocante ou de leur naïveté, les victimes «l'ont bien cherché ».

$\mathrm{Au}$ contraire, les photos représentant des sexes d'hommes (dick pics) où les contenus multimédias montrant des hommes sexuellement actifs suscitent, de la part des internautes, un continuum de réactions s'étendant des plaisanteries inoffensives (dans certaines cultures adolescentes, ces plaisanteries ciblent les jeunes hommes dont les avances sous la forme de dick pics sont déclinées par la destinataire, voire se soldent par un franc rejet) à une certaine admiration. Ces attitudes s'avèrent sans égale mesure avec la sévère réprobation que subissent les cibles féminines de revenge porn. En témoignent les nombreux cas de suicides - analysés dans plusieurs articles consécutifs à des actes de revenge porn, accompagnés de cyber harcèlement de la part d'internautes qui participent à l'humiliation et au lynchage de la victime (Dodge, 2016 ; McGlynn et al, 2017 ; Ricciardelli et Adorjan, 2018).

Ce lynchage numérique entend obtenir une forme de «légitimation» par la construction réifiante de ses victimes. Ces dernières font l'objet de nombreuses injures - « salope ", " pute », etc. - qui les enferment dans des identités dévalorisantes selon un processus visant à justifier l'opprobre publique qu'elles subissent (Dodge, 2016). Ainsi, le continuum d'actes malveillants auxquels le concept médiatique de revenge porn fait référence réaffirme le double standard sexuel qui valorise une sexualité masculine ostensiblement active (Couchot-Schiex et al., 2016) et réprouve sans ménagement l'autonomie et la liberté sexuelle des femmes. 


\section{Les cyber violences conjugales}

Dans la litterature française, les chercheur-e-s du centre Hubertine Auclert ont réalisé en 2018 un rapport sur les cyber violences conjugales. Leur enquête par questionnaires révèle que les cyber violences et les violences physiques constituent les deux facettes indissociables d'un même phénomène. Leur étude démontre également que les principaux actes constitutifs de ce phénomène sont le cyber contrôle - contrôler les déplacements et les fréquentations de la partenaire en la sollicitant constamment par mail, par SMS, etc. - le cyber harcèlement, la cyber surveillance via les technologies GPS ou les logiciels espions, la cyber violence administrative - interdire l'accès au site internet de la sécurité sociale, confisquer les codes permettant d'accéder à un compte bancaire en ligne, etc. - et, enfin, les cyber violence sexuelles. Les auteur-e-s mentionnent également les formes de contrôle et de harcèlement que les conjoints violents perpétuent au moyen des téléphones et/ou des réseaux sociaux des enfants du couple.

Les différentes pratiques concourant à isoler la victime de ses proches, afin d'exacerber l'emprise du conjoint violent, ont été documentées dans la littérature scientifique (Hirigoyen, 2005). Le numérique accroît les moyens d'exclusion et d'isolement qui peuvent être mis en œuvre par les agresseurs. À titre d'exemple, ces derniers peuvent pirater les comptes (Facebook, Twitter, etc.) de leur conjointe et harceler ses « amis » en son nom, afin de ternir durablement sa réputation. Les travaux scientifiques sur cette thématique mettent également en exergue les tactiques consistant à rendre public des photos intimes et des contenus embarrassants, soit pour s'adonner à diverses formes de chantage, soit pour mobiliser des alliés et inciter d'autres utilisateurs à participer au cyber stalking et au harcèlement. Molly Dragiewicz et ses collègues (2017) décrivent la manière dont un acte de revenge porn survenant dans le cadre d'une relation de couple peut devenir viral et se transformer en campagne de haine contre la victime. Ce passage du privé au public est facilité, en outre, par les pratiques de doxxing qui désignent la diffusion, sur des sites comme 4chan, Reddit ou des plateformes plus populaires, d'informations personnelles sur la personne ciblée numéro de téléphone, adresse, codes internet, etc. - afin que des internautes s'en saisissent pour perpétrer, à leur tour, des actes hostiles.

\section{Les campagnes de dénigrement ciblant des personnalités médiatiques et politiques}

Dans la littérature scientifique actuelle, un certain nombre de recherches visent à mieux comprendre les phénomènes de harcèlement collectif et d'attaques perpétrés par des groupes d'internautes. Ces travaux étudient les campagnes de dénigrement 
menées contre des personnalités issues de la culture populaire (des actrices), des professionnelles des médias qui revendiquent leurs convictions féministes dans l'espace public numérique (des journalistes, des bloggeuses, etc.) ainsi que des personnalités politiques.

Ces actes misogynes, commis par des groupes, présentent plusieurs caractéristiques communes. Ils sont répétés sur une longue période - par exemple, Anita Sarkeesian, bloggeuse et critique de jeux vidéo, recevait, au point culminant de son harcèlement, une centaine de tweets insultants par jour, ainsi que des menaces de viol et de mort (Benton-Greig et al., 2018) - et s'intensifient avec le temps. Les remarques méprisantes, les insultes et les menaces de viols font progressivement le lit d'expressions de haine plus graves. Les agresseurs d'Anita Sarkeesian avaient également mis au point un jeu vidéo intitulé beat up Anita qui permettait aux joueurs potentiels de gagner des points en frappant le personnage virtuel représentant la vidéo bloggeuse. À la fin du jeu, la récompense consistait, pour le joueur, à contempler le visage tuméfié et ensanglanté d'Anita (Fox et Tang, 2014). Dans la même veine, Zoé Quinn a reçu des menaces physiques et a été contrainte de fuir son domicile lorsqu'elle s'est rendue compte que son adresse postale avait été rendue publique sur Internet (Jane, 2016; Mortensen, 2016).

Le travail mené par Caitlin Lawson (2018) incite à adopter une approche intersectionnelle pour étudier les manifestations de haine en ligne. L'auteure utilise le néologisme « misogynoir » pour désigner le mélange de violence misogyne et raciste que peuvent potentiellement subir les femmes issues de minorités ethniques. Elle étudie l'exemple de l'actrice afro-américaine Leslie Jones, qui a subi une campagne de harcèlement particulièrement virulente en réaction à son rôle dans le remake du film Ghostbusters, sorti en 2016. Outre les multiples insultes et menaces de viol, Leslie Jones a reçu des photos d'elle souillées par des tâches de sperme ainsi que des images associant son nom à des photos de gorilles.

41 Dans la littérature française, l'analyse d'un ensemble de polémiques qui se sont déroulées sur Twitter a permis de mettre au jour la manière dont certaines personnalités politiques cristallisent un mélange de haine misogyne et raciste. Dans le contexte politique de 2014, marqué par la tentative de mise en place des $A B C D$ de l'égalité et de la fausse rumeur concernant l'enseignement obligatoire de l'arabe dans les écoles, l'ancienne ministre de l'Éducation Najat Vallaud Belkacem a fait l'objet de nombreux commentaires dépréciateurs sur les réseaux sociaux. À travers l'analyse d'un corpus de tweets, Virginie Julliard (2018) démontre que les discours de haine construisent l'ancienne Ministre comme l'incarnation de la double menace de féminisation et d'islamisation de la société française. Il en va de même pour l'ancienne Garde des Sceaux Christiane Taubira, accusée de mettre en œuvre une réforme pénale "molle» (Sebbah et al., 2017), la mollesse symbolisant à la fois un stéréotype dévalorisant associé au féminin (qui s'oppose au «dur » masculin) et aux «autres » racialisés.

42 Ainsi, cet état de l'art suggère que les manifestations de misogynie sont façonnées par le contexte dans lequel elles s'inscrivent. Si notre corpus ne permet pas d'identifier des pratiques généralisables à toute forme de violence numérique, il est toutefois possible de pointer certains dénominateurs communs aux expressions de dérision et de haine misogynes. Les travaux sur le cyber harcèlement, le revenge porn, la violence conjugale ou les campagnes de dénigrement démontrent que des formes de misogynie à l'œuvre 
dans le cadre d'une discussion interpersonnelle peuvent rapidement se muer, dans l'espace public numérique, en violence collective. De plus, les expressions de haine ne demeurent pas confinées à l'intérieur de chaque plateforme, mais semblent circuler de manière fluide entre elles. Les trolls ${ }^{2}$ misogynes peuvent, par exemple, lancer des appels au «harcèlement ", en précisant les coordonnées de la victime, sur des sites comme Reddit ou 4chan, dont la modération quasi inexistante autorise, en leur sein, des débordements langagiers (Mortensen, 2016 ; Marwick et Caplan, 2018). Les internautes que les trolls sont parvenus à rallier et à mobiliser peuvent ensuite passer à l'action sur des plateformes plus populaires comme Twitter, Facebook ou YouTube (Massanari, 2017).

Que la haine misogyne soit exprimée par l'intermédiaire de l'humour, du sarcasme, de l'injure ou de contenus multimédias dégradants, elle a pour conséquence de réduire les femmes ciblées à des stéréotypes, à des corps ou à des objets sexuels. En ce sens, les différentes manifestations de haine misogynes ont un effet « disciplinant " (Cole, 2015) et contribuent à réactualiser un ensemble de croyances sexistes et hétéronormatives constitutives d'un ordre symbolique genré analysé, notamment, par Marie-Joseph Bertini (2009). Si le cyber harcèlement et le revenge porn réaffirment le «double standard » contraignant en matière de mœurs sexuelles, les campagnes de dénigrement renvoient les femmes aux marges de l'espace public. En somme, les actes plus ou moins intenses de cyber misogynie sont mobilisées à des fins idéologiques et permettent aux instigateurs de "remettre chacune et chacun à la 'place' qui lui est assignée dans le système de genre » (Couchot-Schiex et al., 2016).

\section{Acteurs, modèles économiques et cadrage législatif relatifs à la cyber misogynie}

La haine misogyne ne semble pas avoir pour unique but de causer du tort à des cibles féminines stéréotypées et fantasmées. Elle présente également des bénéfices pour le groupe d'instigateurs. Selon Joanne Garde-Hansen et Kristyn Gorton (2013), les pratiques consistant à partager, entre internautes, des photos et des vidéos de femmes nues semblent avoir pris le relais des traditions qui réduisaient le corps des femmes à une monnaie d'échange assurant la stabilité des relations entre différentes communautés. En outre, les auteures associent ces pratiques au phénomène de la rumeur. L'échange de contenus multimédia représentant des corps dénudés faciliterait l'expression d'émotions telles que la jalousie, l'envie, la peur, l'anxiété, etc., au travers desquelles les liens entre internautes seraient consolidés.

\section{Les tenants de la masculinité geek}

Selon Marla Mantilla (2013), les «trolls » et, plus largement, les internautes perpétrant de manière anonyme des actes de cyber misogynie seraient, majoritairement, des hommes blancs, issus de milieux privilégiés et se revendiquant d'une appartenance à la culture geek. Michael Salter (2017) précise que la masculinité geek s'est construite comme une identité alternative à la masculinité hégémonique.

Aux prouesses sportives et sexuelles, aux traits de caractères virils propres à la masculinité hégémonique s'opposerait la vivacité d'esprit et l'expertise technologique associées à la masculinité geek. Marion Coville démontre que les récits et mythes qui 
structurent la culture geek dissipent les stigmates dépréciateurs attribués aux férus d'informatique et de jeux vidéo en construisant « une représentation hégémonique de la masculinité dans l'association à un univers de combat, aux sciences et aux technologies " (2014: 3). Michael Salter ajoute que cette identité alternative s'avère fragile, car elle implique, pour assurer sa cohésion et sa pérennité, la réaffirmation continuelle de la supériorité des compétences informatiques et technologiques des hommes en comparaison à la supposée inaptitude des femmes dans ces domaines.

Michael Salter (2017) et Adrienne Massanari (2017) appréhendent le Gamergate ainsi que d'autres phénomènes de cyber harcèlement collectifs comme une dévalorisation du féminin nécessaire à la cohésion et à la persistance de la culture masculine geek, et comme un backlash en réaction à la participation croissante des femmes à la sphère du jeu vidéo et au cyber espace en général, considéré par certains comme une chasse gardée masculine.

Dans leurs discours, les instigateurs du Gamergate justifient la violence de leurs attaques en se présentant comme les «victimes" des «social justice warriors» qui, en militant en faveur d'une plus grande diversité tant au niveau des représentations que des professionnel-le-s en charge de la conception des jeux, chercheraient selon eux à « aseptiser » et détruire l'univers traditionnel (exclusivement masculin) du jeu vidéo (Mortensen, 2016).

\section{Les sympathisants des mouvements masculinistes}

Plusieurs travaux de notre corpus suggèrent que la cyber misogynie serait en partie liée aux différents acteurs qui gravitent autour de la manosphere. Les groupes masculinistes - porteurs d'un ensemble de discours favorables à la suprématie masculine - ont investi le cyber espace depuis la fin des années 1990 et diffusent leur idéologie sur des blogs et des sites web Dupuis-Deri (2018). Sur ces plateformes, les masculinistes véhiculent un certain nombre de croyances selon lesquelles : «les inégalités de genre ayant disparu, les nouveaux droits des femmes seraient aujourd'hui un privilège tandis que les hommes, en perte de repères, seraient en situation de discrimination dans les sphères conjugales, professionnelles...» (Kunert, 2017: 94) Les membres de ces groupes récupèrent un ensemble de concepts issus des courants de pensée critiques et féministes qu'ils investissent pour défendre les privilèges qu'ils estiment menacés. Ainsi, ces groupes se présentent comme des «contre-publics subalternes» luttant contre une prétendue « hégémonie féministe ».

La diffusion de leur idéologie s'effectue également via des commentaires sexistes et des insultes misogynes sur les forums de discussion et les réseaux socionumériques (Schmitz et Kaykaz, 2016). Marwick et Caplan (2018) constatent que les instigateurs du Gamergate ont largement eu recours à la stratégie de "l'inversion ", amplement utilisée et prônée par les groupes masculinistes, pour légitimer et perpétrer impunément leurs attaques : ils considéraient que leur violence numérique était justifiée, dans la mesure où, d'après eux, ils ne faisaient que se défendre face à des féministes " bourreaux », des « agresseur-e-s » qui les « harcelaient ». 


\section{Des acteurs issus du spectre de l'alt-right}

51 Jenny Sunden et Susanna Paasonen (2018) estiment que, dans les pays nordiques la misogynie en ligne serait étroitement liée à d'autres formes de cyber violences homophobes, racistes et anti-immigration perpétrées principalement par des sympathisants d'extrême droite. Dans les pays anglo-saxons, les membres de l'alt-right ont investi le cyber espace dès la fin des années 1990 afin d'accroître leur visibilité, de rallier des sympathisants et de diffuser leur idéologie (Lawson, 2018).

Theodore Koulouris (2018) estime qu'au travers des actes misogynes, ces groupes d'internautes issus de l'alt-right défendent une vision naturaliste et hiérarchisée de la différence des sexes et se montrent enclins à persécuter ceux qui refuseraient de relayer leur vision des identités et des rapports de genre. L'auteur précise également que les actes misogynes, homophobes et racistes commis par ces internautes témoignent de leur hostilité envers les idéologies et les formations économicopolitiques qui consacrent l'égalité des droits ainsi qu'un partage équitable des ressources économiques. Selon Koulouris (2018) et Adriennen Massanari (2018), les formes de mépris, d'humiliation et d'insulte envers les femmes en ligne s'inscriraient dans le prolongement d'un ensemble de tactiques mises en œuvre par une élite économique soucieuse de préserver son hégémonie face à la montée, perçue comme menaçante, du «socialisme économique » et de valeurs égalitaires dans les pays anglosaxons.

Ainsi, la haine misogyne participerait à la constitution d'un collectif, mobilisé autour de cet affect (Ahmed, 2004) et d'un « objet d'amour » à protéger. Il s'agit ici d'une virilité perçue comme étant " déchue », «bafouée », « abîmée ", et qu'il convient de restaurer par des injures et des campagnes de dénigrement dirigée contre certaines icônes féministes. Cet «objet d'amour" fédère des groupes, aux positions politiques divergentes, qui agissent de concert en vue de réinstaurer une conception essentialiste et asymétrique des identités et rapports sociaux de genre.

Dans la même veine, Christine Servais démontre, dans son analyse de la page Facebook de «Français de Souche » (2017), que la construction d'un objet de haine confère une identité collective et une importante force de mobilisation à des communautés qui ne peuvent pas aisément être regroupées sous la même bannière politique. Ainsi, la haine misogyne entraîne dans son sillage des " publics susceptibles de se constituer en formes collectives de prise de parole» (p.81) ; ces publics disposent d'un pouvoir de nuisance non négligeable dans l'espace public numérique. Si ces groupes n'adhèrent pas aux mêmes idéologies politiques, leurs discours et croyances présentent plusieurs points de convergence, comme en témoigne leur propension à légitimer leur violence en se présentant comme les victimes du "féminisme, » de l'intelligentsia de gauche et, plus globalement, du « politiquement correct» (Slater, 2017; Koulouris, 2018; Massanari, 2018).

55 Ce tour d'horizon des travaux portant sur les instigateurs de misogynie en ligne incite à réactualiser la manière d'appréhender les phénomènes de trolling et de flaming. Dans la littérature académique, le troll désigne un individu qui se complait à perturber une communauté virtuelle en postant des messages délibérément provocants. Comme l'indique Antonio Casilli : « la sanction la plus efficace est l'isolement social de l'usager fautif (...) Ses sollicitations seront ignorées et ses messages haineux, restés sans réponse et sans écho, s'éteindront peu à peu. » $(2010: 322)$. Il faut insister ici sur le fait que dans 
le cadre de la misogynie en ligne, le schéma inverse est à l'œuvre: plusieurs trolls, agissant de manière plus ou moins coordonnée, s'en prennent à une cible en s'adonnant à des actes d'une violence croissante, débordant les frontières d'Internet. Ces remarques incitent à se distancier d'une conception psychologisante du trolling, et à considérer davantage le web comme un nouveau terrain de la "lutte pour le sens" (Hall, 2008) où différents groupes cherchent à véhiculer et à fixer, via des formes de raillerie, de mépris et d'injure, leur conception des identités et des rapports sociaux de genre, de classe et de race.

\section{Les aspects financiers et juridiques de la cyber misogynie}

Outre son rôle dans la constitution d'identités et la consolidation de collectifs, la haine misogyne, exprimée sur Internet, permet à un certain nombre d'acteurs économiques de prospérer. Le "travail affectif» (Alloing et Pierre, 2017) réalisé au sein des plateformes numériques vise à exploiter les affects négatifs associés à la cyber misogynie afin de les monétiser de plusieurs manières. Adrienne Massanari (2017) explique que sur les forums de discussion des sites comme Reddit ou 4chan, connus pour leur grande permissivité, les thématiques relatives à la violence génèrent un surcroit de trafic et, par conséquent, d'importants revenus publicitaires. Ce serait pour cette raison que les administrateurs de ces sites se montreraient réticents à modérer les contenus misogynes dépréciateurs.

Dans la même veine, le Gamergate a engendré des bénéfices financiers considérables pour les agresseurs. En effet, la couverture médiatique du phénomène a fait monter en flèche l'audience des vidéos de plusieurs YouTubeurs qui diffusaient et relayaient sur plusieurs plateformes des contenus haineux envers les femmes. Cet accroissement notable du nombre de "vues» s'est traduit, pour les instigateurs, par une nette augmentation de leurs revenus publicitaires (Mortensen, 2016).

Ganaele Langlois et Andrea Slane (2017) ont étudié le modèle économique des sites de revenge porn. Ces plateformes parviennent à monétiser fructueusement les contenus générés par les internautes - photos et vidéos intimes à caractère sexuel - et entraînent dans leur sillage une myriade d'acteurs s'étendant de l'industrie du porno aux entreprises proposant des conseils en e-réputation et ciblant les victimes dont l'image a été durement et durablement ternie par les contenus embarrassants rendus publics par leurs ex-conjoints. Des mouchards («trackers») installés sur ces sites collectent des données sur les utilisateurs qui seront ensuite revendues à l'industrie du porno. De plus, ces plateformes seraient rémunérées en fonction de leur capacité à rediriger leur trafic vers des sites pornographiques payants. Ce modèle économique semble s'avérer lucratif, comme en témoigne la fortune personnelle que s'est constituée Hunter Moore grâce à son site de revenge porn (fermé depuis 2012) IsAnyoneUp.com (Garde-Hansen et Gortin, 2013). Avant sa fermeture en 2012, IsAnyoneUp recevait en moyenne 300000 visiteurs par jour, audience qu'il était aisé de traduire en revenus publicitaires (McGlynn et al., 2017).

Plusieurs travaux anglophones indiquent qu'aux États-Unis, les victimes de revenge porn peinent à faire entendre leur voix et à obtenir réparation dans la mesure où les instigateurs se protègent massivement derrière le $1^{\mathrm{er}}$ amendement de la Constitution relatif à la liberté d'expression. L'impunité des agresseurs est renforcée par la section $230 \mathrm{du}$ Communications Decency Act qui confère aux plateformes un statut 
"d'hébergeur » qui leur permet de décliner toute responsabilité quant aux contenus haineux publiés par les internautes (Tungate, 2014; Sebastian, 2017). Ce constat ne signifie pas que les internautes français-e-s soient mieux protégé-e-s. Comme l'explique Olivier Iteanu, la loi LCEN de 2004 établit une distinction similaire entre un éditeur et un hébergeur, ce dernier étant considéré uniquement comme un intermédiaire technique bénéficiant d'un "régime de responsabilité favorable et dérogatoire au droit commun" (2016: 42) au regard des contenus hébergés. De plus, les conditions générales d'utilisation (CGU) des principales plateformes imposent aux usagers français l'application du droit états-unien, qui protège la « liberté d'expression » au détriment des victimes d'injures et de propos discriminatoires. Concernant le versant technologique, l'anonymat des instigateurs, ainsi que la possibilité de stocker des contenus haineux sur des serveurs basés à l'étranger constituent des obstacles qui freinent considérablement et obstruent les éventuelles poursuites judiciaires.

Ce tour d'horizon suggère que les victimes de cyber misogynie se trouvent enserrées dans un maillage sociotechnique, juridique et économique favorable aux instigateurs. En France, un ensemble de dispositifs juridiques, visant à contribuer à la prévention des phénomènes liés à la cyber misogynie, commence à être élaboré.

61 La «loi Schiappa » approuvée par l'Assemblée Nationale le 3 août 2018 comporte un volet sur la lutte contre le cyber harcèlement en groupe. Cette loi modifie les dispositions "relatives aux délits de harcèlement sexuel et de harcèlement moral » définies par l'article 222-33-2-2 du code pénal ${ }^{3}$. Cette loi sanctionne les agissements hostiles perpétrés par " plusieurs personnes, de manière concertée ou à l'instigation de l'une d'elles » au moyen « d'un support numérique ou électronique ». Plus récemment, la proposition de loi (dite "Avia») contre les contenus haineux sur Internet a été adoptée en première lecture par l'Assemblée Nationale le 9 juillet $2019^{4}$. La loi Avia vise à contraindre les réseaux socionumériques, les plateformes collaboratives et les moteurs de recherche à retirer les contenus illicites, et notamment les violences sexistes et homophobes, signalés par les internautes, dans un délai de $24 \mathrm{~h}$. Le texte prévoit également l'homogénéisation des modes de signalement via la mise en place d'une procédure de notification uniforme.

Il convient également de souligner les contributions notables des acteurs institutionnels et associatifs qui proposent des dispositifs de sensibilisation, de formation et de prévention. Citons, à titre d'exemple, l'ONG RespectZone ${ }^{5}$, fondée par le juriste Philippe Coen en 2014 afin de promouvoir la civilité et le respect dans les échanges numériques. Les membres de cette ONG ont mis en place un label, une charte, ainsi que des outils de formation relatifs à la modération des contenus haineux. Le centre Hubertine Auclert, dont les travaux ont été cités dans le présent article, a élaboré deux rapports sur les cyber violences sexistes et propose un ensemble de ressources documentaires sur ce thème. Enfin, nous pouvons mentionner l'apport essentiel de certaines associations féministes, comme Femmes Solidaires ${ }^{6}$ ou l'association Féministes contre le cyber harcèlement ${ }^{7}$, qui contribuent, par leurs actions, à la déconstruction des stéréotypes de genre dévalorisants et à la diffusion de discours alternatifs aux idéologies patriarcales, racistes et misogynes amplement véhiculées sur la toile. 


\section{Conclusion} fois racistes, misogynes, antisémites, homophobes et transphobes, qui trouvent sur Internet le moyen de constituer des collectifs violents, qui s'en prennent à des victimes, parfois en toute impunité, sans que les réactions des plateformes ou des pourvoyeurs de technologies ne soient à la hauteur des méfaits constatés. S'agissant plus particulièrement de misogynie, les effets peuvent être particulièrement graves et concrets. Subir ou être témoin de cyber misogynie représente, pour Kristi Cole (2015), une forme de «discipline » visant à écarter les femmes de l'espace public numérique. Dans cette veine, une enquête IPSOS MORI ${ }^{8}$ commanditée par Amnesty International a été réalisée auprès d'un échantillon de femmes provenant de 8 pays (Danemark, Italie, Nouvelle-Zélande, Pologne, Espagne, Suède, Royaume-Uni et États-Unis.) Cette étude révèle que $76 \%$ des victimes de violences sexistes en ligne auraient "modifié la manière d'utiliser ces plateformes ». De plus, $32 \%$ d'entre elles auraient cessé d'exprimer leur opinion sur Internet. Ainsi, loin de favoriser la diversité des opinions, les garde-fous juridiques visant, à l'origine, à garantir la liberté d'expression sur Internet ont entrainé l'impunité des discours de haine et ont participé à l'exclusion des groupes sociaux et des minorités traditionnellement tenus à l'écart de l'espace public (Lumsden et Morgan, 2017 ; Gardiner, 2018).

67 Ce retrait de l'espace public numérique, par crainte de subir des représailles, entraînerait des conséquences notables sur le plan professionnel (Megarry, 2014 ; Jane, 2016). Cette stratégie de protection empêcherait les femmes de bénéficier d'opportunités professionnelles et affaiblirait leur réseau de relations. Enfin, pour celles dont la prise de parole dans l'espace public représente le métier (journalistes, bloggeuses etc.), le retrait du web aurait pour effet immédiat une perte dramatique de revenu. Au regard de l'ampleur de l'usage des TIC dans de nombreux emplois actuels, Emma Jane (2018) estime que la cyber misogynie représente une menace potentielle pour n'importe quelle femme active. 

termes psychosociaux à l'échelle de la population féminine, stigmatisée et pour laquelle peu de politiques publiques de prévention sont menées, notamment en France. La cyber misogynie provoquerait des conséquences délétères en termes de santé mentale et physique. Comme le souligne Emma Jane (2016), les femmes victimes de campagnes de haine et de cyber harcèlement doivent composer avec de l'anxiété, du stress post traumatique et de la dépression. En France, un rapport commandité par le Haut Conseil à l'Égalité corrobore ces résultats et précise que le risque de se suicider est « 3,17 fois plus élevé lorsque l'on est victime de harcèlement sur les réseaux sociaux (Bousquet et al., $2017: 39) »$.

En conclusion, les études consultées et analysées montrent, à la fois, que les pratiques misogynes en ligne produisent tant une mise en visibilité et une intensification du phénomène de misogynie qu'une instrumentalisation économique de celui-ci. De tels actes servent de nouvel étendard idéologique à des groupes radicaux, qui trouvent peu de contrepoids à leur action, notamment judiciaires. Les conséquences en termes de discrimination, de vulnérabilisation et de victimisation sont importantes et les lanceurs et lanceuses d'alerte, sur ce sujet, sont la proie d'opérations de lynchage numérique, les conduisant régulièrement à devoir changer de vie, d'environnement, d'activités professionnelles.

Pour Axel Honneth (2008), la reconnaissance sociale constitue « la condition normative de toute activité communicationnelle " et permet de développer trois catégories de rapports positifs à soi : la confiance en soi, le respect et l'estime de soi. Dans le contexte que nous venons de décrire, et notamment les pratiques qui ont fait l'objet des études citées, les conditions d'une reconnaissance sociale des pratiques des femmes en ligne sont loin d'être acquises. L'humour stéréotypé, les humiliations et les insultes en ligne sont autant de déni de reconnaissance, de mépris, qui suscitent des émotions négatives et peuvent légitimement relever du cyber harcèlement, comme les derniers développements autour d'un groupe de journalistes masculins aux actions misogynes, réunis dans un groupe Facebook, «la ligue du LOL» le prouvent aujourd'hui. Les travaux montrent aussi que les frontières entre misogynie en ligne et cyber harcèlement sont ténues, tout comme le continuum de violence discriminatoire envers les personnes de groupes minoritaires, racisées, discriminées pour des raisons de genre ou religieuses. Les terrains potentiels de recherche sur ces questions, qui demandent des précautions particulières, étant donné les témoignages recueillis des chercheurs ou chercheuses ayant été harcelés pour s'être intéressés à ces sujets, sont nombreux et encore trop peu investigués.

\section{BIBLIOGRAPHIE}

AHMED Sara (2004), « Affective economies », Social text. vol. 2, n 22, p. 117-139. 
Allard Laurence, Camille Alloing, Mariannig Le BeCheC et Julien PierRe (2017), « Introduction. Les affects numériques ", Revue française des sciences de l'information et de la communication [en ligne]. $\mathrm{n}^{\circ}$ 11. Disponible à l'adresse : http://journals.openedition.org/rfsic/2870.

ALLOING Camille et Julien PIERRE (2017), Le Web affectif. Une économie numérique des émotions. Brysur-Marne : INA Éditions.

Centre Hubertine Auclert (2018), Cyber-violences conjugales. Recherche-action menée auprès de femmes victimes de violences conjugales et des professionnel-le-s les accompagnant. Paris.

BARAK Azy (2005), « Sexual harassment on the Internet », Social Science Computer Review. vol. 23, $\mathrm{n}^{\circ} 1$, p. 77-92.

Benton-Greig Paulette, Dhakshi Gamage et Nicola Gavey (2018), « Doing and denying sexism : online responses to a New Zealand feminist campaign against sexist advertising ", Feminist media studies. vol. 18, $\mathrm{n}^{\circ}$ 3, p. 349-365. DOI 10.1080/14680777.2017.1367703.

BERTINI Marie-Josephe (2009), Ni d'Ève ni d'Adam. Défaire la différence des sexes. Paris : Max Milo Éditions.

BERTINI Marie-Josephe (2012), « Genre et médias à l'épreuve de l'affaire DSK. Réflexions sur le commentaire en ligne, nouvel espace de construction de l'inégalité des sexes » Sciences de la société. $\mathrm{n}^{\circ} 83$, p. 54-64.

BERTINI Marie-Joseph (2016), « Genre 2.0. Le web, un champ structuré en domination. Vers une économie politique des pratiques langagières en ligne ", Genre en séries : cinéma, télévision, média. $\mathrm{n}^{\circ} 3$, p. 23-44.

BLAYA Catherine (2018), « Le cyberharcèlement chez les jeunes », Enfance. n 3, p. 421-439.

BLAYA Catherine (2019), Cyberhaine. Les jeunes et la violence sur Internet. Paris : Nouveau Monde éditions.

Boonthanom Ranida (2004), Computer-Mediated Communication of Emotions: A Lens Model Approach. Doctoral dissertation in management information systems. Florida : Florida State University. Bousquet Danielle, Edouard DurAnd, Ernestine RonAI, Alice GAYRAud, et Claire GuiRAud (2017), En finir avec l'impunité des violences faites aux femmes en ligne : une urgence pour les victimes. Haut Conseil à l'Égalité entre les femmes et les hommes.

CASILLI Antonio A. (2010), Les Liaisons numériques. Vers une nouvelle sociabilité. Paris : Seuil.

CASSIDY Wanda, Margaret JACKSON et Chantal FAUCHER (2016), « Gender differences and cyberbullying towards faculty members in higher education », Cyberbullying across the globe. Gender, Family and Mental health : Springer. p. 79-98.

CERVUlle Maxime et Virginie JULLIARD (2018), « Le genre des controverses : approches féministes et queer ", Questions de communication. $\mathrm{n}^{\circ} 33$, p. 7-22.

CoLE Kristi K. (2015), « “It's like she is eager to be verbally abused” : Twitter, trolls and (en)gendering disciplinary rhetoric ", Feminist media studies. vol. 15, nº 2, p. 356-358.

COUCHOT-SCHIEX Sigolène, Benjamin MoIGNARD et Gabrielle RICHARD (2016), Cybersexisme : une étude sociologique dans des établissements scolaires franciliens. Paris. Centre Hubertine Auclert.

Coville Marion (2014), « Créateurs de jeux vidéo et récits de vie : la formation d'une figure

hégémonique ", Revue française des sciences de l'information et de la communication [en ligne]. $\mathrm{n}^{\circ} 4$. DOI 10.4000/rfsic.763. Disponible à l'adresse : http://journals.openedition.org/rfsic/763. 
CULNAN Mary J. et M. Lynne MARKUS (1987), « Information technologies », Handbook of organizational communication: An interdisciplinary perspective. Thousand Oaks : Sage Publications, Inc. p. $420-443$.

DAUdigeos T. et al. (2014), « Bouc-émissaires, lynchages médiatiques et contestation des pratiques irresponsables des firmes multinationales ", Revue de l'organisation responsable. vol. 9, n 2, p. 46-59

Daumas Maurice (2017), Qu'est-ce que la misogynie ? Paris : Les éditions Arkhê.

DEKESEREDY Walter S. et Martin D. SCHWARTZ (2016) « Thinking Sociologically About Image-Based Sexual Abuse : The Contribution of Male Peer Support Theory ", Sexualization, Media, \& Society. vol. 2, n 4, DOI 10.1177/2374623816684692.

DoDge Alexa (2016), « Digitizing rape culture : Online sexual violence and the power of the digital photograph », Crime, Media, Culture. vol. 12, n 1, p. 65-82. DOI 10.1177/1741659015601173.

Dragiewicz Molly, Jean Burgess, Ariadna Matamoros-Fernandez, Michael Salter, Nicolas P. Suzor, Delanie WOODLOCK, et Bridget HARRIS (2018), « Technology facilitated coercive control : domestic violence and the competing roles of digital media platforms ", Feminist media studies. vol. $18, \mathrm{n}^{\circ} 4$, p. 609-625. DOI https://doi.org/10.1080/14680777.2018.1447341.

DRAKETT Jessica, Bridgette RicketT, Katy DAy et Kate Milnes (2018), « Old jokes, new media - online sexism and constructions of gender in Internet memes ", Feminism \& Psychology. vol. 28, n 1 , p. 109-127.

DUPUIS-DERI Francis (2018), La Crise de la masculinité. Autopsie d'un mythe tenace. Montréal : Les éditions du remue-ménage.

FelmLEe Diane et Robert FARIS (2016), « Toxic Ties : Networks of Friendship, Dating, and Cyber Victimization ", Social Psychology Quarterly. vol. 79, n³, p. 243-262.

DOI 10.1177/0190272516656585.

Fox Jesse, Carlos CRUZ et Ji Young LeE (2015) « Perpetuating online sexism offline : anonymity, interactivity, and the effects of sexist hashtags on social media ", Computers in Human Behavior. vol. 52, p. 436-442.

Fox Jesse et Wai Yen TANG (2014), «Sexism in online video games : The role of conformity to masculine and social dominance orientation ", Computer in Human Behavior. vol. 33, p. 314-320. DOI 10.1016/j.chb.2013.07.014.

FRAME Alexander (2017), « Personnel politique et médias socionumériques : nouveaux usages et mythes 2.0 », La Communication politique. Paris : CNRS Éditions.

FRIEDMAN Raymond A. et Steven C. CURRALL (2003), « Conflict escalation : dispute exacerbating elements of e-mail communication », Human Relations. vol. 56, n 11, p. 1325-1347.

GARDE-HANSEN Joanne et Kristyn GORTON (2013), Emotion online : theorizing affect on the internet : Palgrame/Mc Millan publishing.

GARDINER Becky (2018), « “It's a terrible way to go to work :" what 70 million readers' comments on the Guardian revealed about hostility to women and minorities online », Feminist Media Studies. vol. $18, \mathrm{n}^{\circ}$ 4, p. 592-608. DOI 10.1080/14680777.2018.1447334.

GIRARD René (2015). Le Bouc émissaire. Paris, Livre de Poche Biblio Essais, [Grasset, 1982].

HALL Stuart (2008), Identités et cultures : politiques des cultural studies. Paris : Éditions. Amsterdam. 
HARDAKER Claire et Mark MCGLASHAN (2016), « “Real men don't hate women” : Twitter rape threats and group identity », Journal of Pragmatics. vol. 91, p. 80-93.

HIRIGOYEN Marie-France (2005), Femmes sous emprise. Les ressorts de la violence dans le couple. Paris : Oh ! Éditions.

HONNETH Axel (2007), La Réification. Petit traité de théorie critique. Paris : Gallimard.

HoNNETH Axel (2008), La Société du mépris : vers une nouvelle théorie critique. Paris : La découverte.

ITEANU Olivier (2016), Quand le digital défie l'État de droit. Paris : Editions Eyrolles.

JANE Emma A. (2016), Misogyny Online: A Short (and Brutish) History. Thousand Oaks, CA : SAGE Publications Ltd.

JANE Emma A. (2018), « Gendered cyberhate as workplace harassment and economic vandalism », Feminist Media Studies. vol. 18, nº 4, p. 575-591. DOI 10.1080/14680777.2018.1447344.

JOUET Josiane (2003), « Technologies de communication et genre », Réseaux. vol. 120, nº 4, p. 53-86. JULLIARD Virginie (2014), « Un mode d'appropriation des gender studies par les sciences de l'information et de la communication : la sémiotique du genre ", Questions de communication. $\mathrm{n}^{\circ} 25$, p. 223-243. DOI 10.4000/questionsdecommunication.9028.

JULLIARD Virginie (2016), « \#Theoriedugenre : comment débat-on du genre sur Twitter? », Questions de communication. $\mathrm{n}^{\circ}$ 30. DOI 10.4000/ questionsdecommunication.10744.

JULLIARD Virginie (2017), « “Théorie du genre”, \#theoriedugenre : stratégies discursives pour satisfaire la « différence des sexes » des objets de débat ", Études de communication. n 48. DOI $10.4000 /$ edc.6811.

JULLIARD Virginie (2018), «L'idéologie raciste en appui aux discours antiféministes : les ressorts émotionnels de l'élargissement de l'opposition à la 'théorie du genre' à l'école sur Twitter ", Cahiers du Genre. vol. 65, n 2, p. 17-39.

KELLER JOHNSON Lauren (2002), « Does e-mail escalate conflict? The idiosyncratic aspects of electronic mail can obviate resolution », MIT sloan management review. $\mathrm{n}^{\circ} 15, \mathrm{p} .14-15$.

KIMMEL Michael (2009), GuyLand: The perilous world where boys become men. Understanding the critical years between 16 and 26. New York : HarperCollins.

KoULOURIS Theodore (2018), «Online misogyny and the alternative right : debating the undebatable ", Feminist media studies. vol. 18, n 4, p. 750-761. DOI 10.1080/14680777.2018.1447428.

KROHN Franklin B. (2004), « A generational approach to using emoticons as nonverbal communication », Technical writing and communication. vol. 34, $\mathrm{n}^{\circ} 4$, p. 321-328.

KUNERT Stéphanie (2017), « Stratégies de légitimation et configurations discursives de la "cause des hommes" ", Études de communication. Langages, information, médiations. n 48, p. 91-110. DOI 10.4000/edc.6802.

LANGLOIS Ganaele et Andrea SLANE (2017), «Economies of reputation : the case of revenge porn », Communication and critical/cultural studies. vol. 14, $\mathrm{n}^{\circ} 2$, p. 120-138.

DOI 10.1080/14791420.2016.1273534.

LAWSON Caitlin E. (2018) « Platform vulnerabilities : harassment and misogynoir in the digital attack on Leslie Jones ». In : Information, Communication \&Society. vol. 21, n 6, p. 818-833. DOI 10.1080/1369118X.2018.1437203. 
LUMSDEN Karen et Heather MORGAN (2017), « Media framing of trolling and online abuse : silencing strategies, symbolic violence, and victim blaming », Feminist Media Studies. vol. 17, nº 6, p. 926-940. DOI 10.1080/14680777.2017.1316755.

MANTILLA Marla (2013), « Gendertrolling : Misogyny adapts to new media », Feminist Studies. vol. $39, \mathrm{n}^{\circ} 2$, p. 563-570.

MANTZIARI Despoina (2018), « Sadistic scopophilia in contemporary rape culture : I Spit On Your Grave (2010) and the practice of "media rape" ", Feminist Media Studies. vol. 18, n 3, p. 397-410. DOI 10.1080/14680777.2017.1367700.

MARKUS Lynne M. (1994), «Finding a Happy Medium : Explaining the Negative Effects of Electronic Communication on Social Life at Work », ACM Transactions on information systems. vol. 12, n² 2, p. 119-149. DOI 10.1145/196734.196738.

MARWICK Alice E. et Robyn CAPLAN (2018), « Drinking male tears : language, the manosphere, and networked harassment ", Feminist Media Studies. vol. 18, n 4, p. 543-559.

DOI 10.1080/14680777.2018.1450568.

MASSANARI Adrienne (2017), « \#Gamergate and The Fappening: How Reddit's algorithm, governance, and culture support toxic technostructures ", New media \& society. vol. 19, $\mathrm{n}^{\circ} 3$, p. 329-346. DOI https://doi.org/10.1177/1461444815608807.

MASSANARI Adrienne (2018), " Rethinking research ethics, power, and the risk of visibility in the Era of the « Alt-Right » gaze », Social media \& society. p. 1-9.

MCCORMICK Naomi et John LeONARD (1996), « Gender and Sexuality in the Cyberspace Frontier », Women \& Therapy. vol. 19, n 4, p. 109-119. DOI 10.1300/J015v19n04_13.

McGlynn Clare, Erika RACKLEY et Ruth Houghton (2017), « Beyond 'Revenge Porn' : The Continuum of Image-Based Sexual Abuse », Feminist Legal Studies. vol. 25, n 1, p. 25-46. DOI 10.1007/ s10691-017-9343-2.

MEGARRY Jessica (2014), « Online incivility or sexual harassment ? Conceptualizing women's experiences in the digital age ", Women's Studies International Forum. vol. 47, p. 46-55.

MEHRABIAN Albert (1972), Nonverbal communication. Chicago : Aldine-Atherton.

MERCIER Arnaud (2018), «L'omniprésence des postures humoristiques sur Twitter », Le Sens de l'humour. Style, genres, contextes. Louvain : Academia.

MORTENSEN Torill Elvira (2016), « Anger, Fear, and Games : The Long Event of \#GamerGate », Games and Culture. vol. $13, \mathrm{n}^{\circ} 8$, p. 787-806.

MYERS, Carrie-Anne et Helen CowIE (2017) « Bullying at University : The Social and Legal Contexts of Cyberbullying Among University Students ", Journal of Cross-Cultural Psychology. vol. 48, $\mathrm{n}^{\circ} 8$, p. 1172-1182.

PATELLA-REY P. J. (2018), « Beyond privacy : bodily integrity as an alternative framework for understanding non-consensual pornography ", Information, Communication \& Society. vol. 21, $\mathrm{n}^{\circ} 5$, p. 786-791. DOI 10.1080/1369118X.2018.1428653.

Pfaff Mark S. et Afarin PIRZADEH (2017), « Multi-methodological approaches for studying emotion in computer-mediated communication », Work pressures : new agendas in communication. New York : Routledge. p. 147-162.

QUEMENER Nelly (2018), “ “Vous voulez réagir ?”. L'étude des controverses médiatiques au prisme des intensités affectives", Questions de communication. vol. 33, n 1, p. 23-41. 
RICCIARDELli Rosemary (Rose) et Michael ADORJAN (2018), « 'If a girl's photo gets sent around, that's a way bigger deal than if a guy's photo gets sent around' : gender, sexting, and the teenage years ", Journal of Gender Studies. vol. 28, n 5, p. 563-577. DOI 10.1080/09589236.2018.1560245.

SALTER Michael (2017), « From geek masculinity to Gamergate : the technological rationality of online abuse ", Crime media culture. vol. 14, p. 247-264.

ScHMITZ Rachel M. et Emily KAZYAK (2016), « Masculinities in Cyberspace : An Analysis of Portrayals of Manhood in Men's Rights Activist Websites ", Social Sciences. vol. 5, n 2, p. 18. DOI 10.3390/socsci5020018.

SEBAStian Melinda (2017), " Privacy and consent : the trouble with the label of "revenge porn" ", Feminist Media Studies. vol. 17, nº 6, p. 1107-1111. DOI 10.1080/14680777.2017.1380428.

SEBBAH Brigitte, Arnaud MERCIER et Romain BADOUARD (2017), « La fabrique des tweets polémiques : l'exemple de la réforme pénale de juin-juillet 2014 », PIGNARD-CHEYnel, Nathalie (éd.), \#info : Commenter et partager l'actualité sur Twitter et Facebook. Paris : Éditions de la Maison des sciences de l'homme. Le (bien) commun. p. 229-268.

SERVAIS Christine (2017), « Scènes médiatiques et arènes de discours 》, Réseaux. vol. 202-203, n² 2, p. 79-121.

SHARIFF Shaheen et Rachel GouIN (2006), « Cyber-dilemmas : Gendered hierarchies, free expression and cyber-safety ", International Conference on Cyber-Safety. Oxford University.

SPROULl Lee et Sara KIESLER (1986), « Reducing social context cues : electronic mail in organizational communication », Management Science. vol. 32, n 11, p. 1492-1512.

STROUD Scott R. (2014), « The dark side of the online self : A pragmatist critique of the growing plague of revenge porn ", Journal of mass media ethics : Exploring questions of media morality. vol. 29, $\mathrm{n}^{\circ}$ 3, p. 168-183. DOI 10.1080/08900523.2014.917976.

SUNDEN Jenny et Susanna PAASONEN (2018), « Shameless hags and tolerance whores : feminist resistance and the affective circuits of online hate ", Feminist media studies. vol. $18, \mathrm{n}^{\circ} 4$, p. 643-656. DOI 10.1080/14680777.2018.1447427.

THOMPSON Laura (2018), “ "I can be your Tinder nightmare" : harassment and misogyny in the online sexual marketplace », Feminism \& Psychology. vol. 28, n 1, p. 69-89.

TUNGATE Allison (2014), « Bare necessities : the argument for a 'revenge porn' exception in Section 230 immunity ». In : Information \& Communications Technology Law. vol. 23, n 2, p. 172-188.

WoodLock Delanie (2017), « The abuse of technology in domestic violence and stalking », Violence against women. vol. 23, $\mathrm{n}^{\circ} 5$, p. 584-602.

\section{NOTES}

1. Les mèmes internet constituent des blagues, montages et des détournements parodiques sous la forme d'images ou de vidéos dont la circulation sur le web peut devenir virale.

2. Le terme «trolls » désigne des internautes qui postent, sur différentes plateformes ou forums de discussion, des messages délibérément provoquants afin de susciter des réactions émotionnelles et de déclencher des rixes verbales (Jane, 2016).

3.

affichTexte.do;jsessionid=A074CC2BE7F0D9E35864944754598AA5.tplgfr25s_2? 
4. http://www.assemblee-nationale.fr/dyn/15/dossiers/alt/lutte_contre_haine_internet

5. www.respectzone.org

6. www.femmes-solidaires.org

7. www.vscyberh.org

8. L'impact inquiétant du harcèlement en ligne à l'égard des femmes, [2017]. Amnesty France [en ligne]. Disponible à l'adresse : www.amnesty.fr/discriminations/actualites/des-femmes-dumonde-entier-font--etat-de-stress-dangoisse

\section{RÉSUMÉS}

Cet article vise à analyser les manifestations de misogynie survenant en ligne à partir d'un corpus de travaux issus de la littérature scientifique française et anglophone. Sont analysées, dans un premier temps, les fonctionnalités techniques du web et des principales plateformes numériques, qui peuvent être utilisées pour matérialiser des affects négatifs et exprimer un continuum de propos violents, s'étendant de l'humour stéréotypé aux menaces d'agression. La deuxième partie de l'analyse est consacrée aux acteurs de la cyber misogynie; elle montre que ces actes tendent à être perpétrés par des groupes d'internautes, souvent radicaux, qui expriment par ce biais un certain nombre de croyances idéologiques. Le dernier point évoqué est celui des modèles économiques qui parviennent à monétiser les manifestations de haine en ligne et assurent ainsi la persistance de ce phénomène.

This paper explores the phenomenon of cyber misogyny and consists of a review of the existing French and English-speaking academic literature. The first part deals with the different ways in which the technical features of the web and mainstream digital platforms are used to materialize negative affects and express a set of violent comments, ranging from sexist humor to rape and aggression threats. In a second part, the actors involved in cyber misogyny are presented. Misogynist banters and slurs tend to be perpetrated by radical groups who are willing to convey their ideological views on the Internet. Finally, the economies fueled by online hate speech targeting women are described.

\section{INDEX}

Mots-clés : cyber misogynie, TIC, cyber harcèlement, affect, communication, violence numérique

Keywords : cyber misogyny, icts, cyber bullying, affect, communication, online violence

\section{AUTEURS}

\section{DELPHINE DUPRÉ}

Delphine Dupré est doctorante en Sciences de l'Information et de la Communication au sein du laboratoire MICA (EA 4426) et ATER à Université Bordeaux Montaigne. Sa thèse porte sur les incivilités numériques en contexte de travail. 


\section{VALÉRIE CARAYOL}

Valérie Carayol est Professeure en Sciences de l'Information et de la Communication à

l'Université Bordeaux Montaigne et responsable d'une équipe de recherche au sein du laboratoire MICA. Ses travaux dans le champ de la communication organisationnelle s'intéressent particulièrement aux questions temporelles et aux phénomènes de communication, qui animent les dynamiques de transformation des organisations. Elle est directrice de la publication de la Revue Communication \& Organisation depuis 2005. 